Gut, 1982, 23, 829-834

\title{
Diagnostic value of serum primary bile acids in detecting bile acid malabsorption
}

\author{
R ALDINI, A RODA, * D FESTI, G MAZZELlA, A M MORSELli, C SAMA, \\ E RODA, N SCOPINARO, and L BARBARA
}

From the Cattedra di Clinica Medica 30, University of Bologna, Policlinico S Orsola, Bologna, Italy, and the Instituto di Scienze Chimische, Facoltà di Farmacia, University of Bologna, Bologna, Italy

SUMMARY Serum cholic and chenodeoxycholic acid conjugates were measured in fasting conditions and after meals in 14 patients with bile acid malabsorption due to ileal resection. Mean serum fasting levels of both primary bile acids did not differ from the controls. After meals, serum cholic acid peaks were lower in patients with ileal resection than in control subjects $(\mathrm{p}<0.001)$, while chenodeoxycholic acid peaks were reduced in colectomised patients $(\mathrm{p}<0 \cdot 01)$. In the sera from patients with ileal resection, the glycine/glycine + taurine ratio for cholic and chenodeoxycholic acid increased $(\mathrm{p}<0.001)$ from morning to evening, and glycine/glycine + taurine ratio for chenodeoxycholic acid was significantly $(\mathrm{p}<0.01)$ different from the controls in the sera collected in the evening. The results are consistent with the concept of a better intestinal conservation of chenyl, mainly of the glycine conjugated form, than of cholylconjugates, in patients with ileal resection; this is probably because of passive absorption in the intestine. The postprandial peaks of serum cholic acid conjugates may therefore be regarded as a test of ileal dysfunction, while peaks of chenodeoxycholic acid conjugates suggest colonic impairment.

Recent studies in $\operatorname{man}^{1-4}$ are consistent with the hypothesis that serum bile acids reflect a balance between their intestinal input and their hepatic clearance from portal blood. Fractional hepatic uptake has been proved to be constant within a wide range of bile acid loads to the liver in animals ${ }^{5}$ and to be unaffected by fasting and postprandial conditions in man. ${ }^{4}$ Intestinal input therefore seems to be the major determinant of serum bile acid levels in the absence of liver disease and differences in the serum profiles of cholic and chenodeoxycholic acids ${ }^{6}{ }^{7}$ result from a different absorptive pattern for the two bile acids. ${ }^{89}$

Diurnal variations of serum cholic ${ }^{10}$ and chenodeoxycholic ${ }^{10}$ acid conjugates have been measured in healthy subjects and in patients with bile acid malabsorption.

These studies showed that in patients with bile acid loss associated with ileal resections serum cholic acid fasting levels are lower than in controls.

Postprandial rises in cholyl conjugates were lower and diminished progressively with each meal, while increases in chenodeoxycholic acid after meals were lower, but constant throughout the day.

Received for publication 26 November 1981
The purpose of the present study was to investigate whether the serum fasting and postprandial levels of bile acids reflect bile acid malabsorption in patients with ileal resection and have clinical applications in the diagnosis of this syndrome.

We measured by radioimmunoassay ${ }^{11}$ the serum levels of the two primary bile acids in fasting and postprandial conditions in healthy subjects and patients with bile acid malabsorption.

We also evaluated the ratio between the two primary bile acids as well as the ratio between their glyco and tauro conjugated forms both in fasting conditions and after meal-stimulated enterohepatic recycling.

\section{Methods}

PATIENTS

Fourteen patients with ileal resection were studied. The length of the resection was evaluated at surgery. No patient presented radiological, histological, or biochemical signs of recurrence of the disease.

Five patients with colectomy for ulcerative colitis and 10 healthy subjects were also studied for control purposes. 
In all patients, bile acid malabsorption had been previously demonstrated by breath analysis and faecal excretion of ${ }^{14} \mathrm{C}$ after oral administration of cholyl $1-{ }^{14} \mathrm{C}$ glycine. ${ }^{12-14}$

The daily faecal excretion of bile acids was evaluated by gas liquid chromatography according to Grundy's technique. ${ }^{5}$ The relevant data are reported in the Table.

The groups were not matched for sex, weight, or age, but there was nothing of note in their physical examinations and they had normal results on conventional liver tests (serum bilirubin, alkaline phosphatase, glutamic oxalacetic and glutamic pyruvic transaminases, $\gamma$-glutamyltranspeptidase values). All the patients and controls had normally functioning gall bladders without gall stones (as shown by oral cholecystograms).

Neither the patients nor the controls had received any drug for at least two weeks before the study.

\section{EXPERIMENTAL DESIGN}

On the morning of the study, a 19-gauge scalp vein needle was inserted into a large antecubital vein; the needle remained in place throughout the study. Five millilitre samples of blood were drawn in fasting conditions. Three solid meals $(300,900$, and 600 calories respectively; $40 \%$ protein, $20 \%$ fat, $40 \%$ carbohydrates) were given at $8.15,12.15$, and 17.15 $h$.

Blood samples were drawn at 08.00, 09.00, 09.30, $10.00,10.30,11.00,12.00,13.00,13.30,14.00$, $14.30,15.00,15.30,16.00,17.00,18.15,18.45$, 19.15 , and $20.00 \mathrm{~h}$. The blood was allowed to clot and the serum was stored at $-20^{\circ} \mathrm{C}$ until bile acid analysis.

The ratio of serum cholyl and chenyl conjugates as well as that of glycine and taurine conjugates of cholic and chenodeoxycholic acid were evaluated only on the serum sample drawn at 8.00 and $20.00 \mathrm{~h}$.

A 12 hour interval was chosen in order to compare the serum primary bile acid pattern in the fasting state and after the meal-stimulated enterohepatic recycling in standardised assay conditions.

\section{RADIOIMMUNOASSAY OF SERUM CHOLIC AND} CHENODEOXYCHOLIC ACID

Serum levels of cholic and chenodeoxycholic acid were evaluated by two specific radioimmunoassays. Studies on cross-reactivity, together with parallelism and recovery studies to assess the reproducibility,

Table Data of patients with ileal resection and colectomy

\begin{tabular}{|c|c|c|c|c|c|}
\hline \multirow[b]{2}{*}{ Patient } & \multirow[b]{2}{*}{$\begin{array}{l}\text { Age (yr) } \\
\text { Sex }\end{array}$} & \multirow{2}{*}{$\begin{array}{l}\text { Length of } \\
\text { resection } \\
(\mathrm{cm})\end{array}$} & \multirow[b]{2}{*}{$\begin{array}{l}\text { Faecal }{ }^{14} C^{*} \\
(\% \text { dose } / 24 h)\end{array}$} & \multicolumn{2}{|c|}{ Faecal bile acids } \\
\hline & & & & $\begin{array}{l}1 \\
\text { (mg/day) }\end{array}$ & 2 \\
\hline BA & $44 F$ & $180 \ddagger$ & 20 & 350 & 260 \\
\hline CG & $45 \mathrm{M}$ & $170 \ddagger$ & 81 & 2466 & 830 \\
\hline MT & $42 \mathrm{~F}$ & $180 \dagger$ & 73 & 1553 & 533 \\
\hline SA & $28 \mathrm{M}$ & $250 \ddagger$ & 35 & 2800 & 1310 \\
\hline GN & $34 \mathrm{M}$ & $280 \ddagger$ & 31 & 4137 & 1947 \\
\hline BL & $41 \mathrm{M}$ & $80 \dagger$ & 33 & 223 & 108 \\
\hline BM & $74 \mathrm{~F}$ & $100 \ddagger$ & 22 & 148 & 100 \\
\hline GI & $32 \mathrm{~F}$ & $100 \ddagger$ & 79 & 1200 & 535 \\
\hline MM & $35 \mathrm{~F}$ & $80+$ & 12 & 406 & 40 \\
\hline BG & $41 \mathrm{M}$ & $100 \ddagger$ & 28 & 300 & 60 \\
\hline AB & $34 \mathrm{M}$ & $80 \dagger$ & 10 & 248 & 140 \\
\hline $\mathrm{CD}$ & $45 \mathrm{~F}$ & $50+$ & 13 & 300 & 130 \\
\hline FF & $28 \mathrm{~F}$ & $100 t$ & 12 & 290 & 185 \\
\hline BB & $29 \mathrm{M}$ & $100 \ddagger$ & 15 & 295 & 140 \\
\hline Mean $\pm S D$ & & & & $1064 \pm 1200$ & $451 \pm 500$ \\
\hline FU & $48 \mathrm{M}$ & & 17 & 280 & 290 \\
\hline $\mathrm{CV}$ & $33 \mathrm{M}$ & & 19 & 220 & 250 \\
\hline DS & $30 F$ & & 23 & 130 & 170 \\
\hline $\mathrm{DE}$ & $32 \mathrm{M}$ & & 27 & 142 & 120 \\
\hline $\mathrm{VZ}$ & $34 \mathrm{~F}$ & & 18 & 130 & 110 \\
\hline Mean $\pm S D$ & & & & $180 \pm 60$ & $188 \pm 76$ \\
\hline Controls & $29 \pm F(6)$ & & $6 \pm$ & $120 \pm$ & $80 \pm$ \\
\hline $\begin{array}{l}(n=10) \\
(\text { mean } \pm S D)\end{array}$ & $4 \mathrm{M}(4)$ & & 4 & 60 & 30 \\
\hline
\end{tabular}

1 : cholic + deoxycholic acid. 2 : chenodeoxycholic + lithocholic acid.

* After oral choly $1-{ }^{14} \mathrm{C}$ glycine $(5 \mathrm{uCi})$.

$\dagger$ Ileum - ascending colon anastomosis.

$\ddagger$ Ileum - transverse colon anastomosis. 
precision, accuracy and applicability of the cholic and chenodeoxycholic acid assays, have already been reported. ${ }^{11}$

\section{DETERMINATION OF GLYCINE AND TAURINE CONJUGATES OF CHOLIC AND \\ CHENODEOXYCHOLIC ACID}

This evaluation was carried out by radioimmunoassay after the glycine and taurine conjugates of the two bile acids had been separated by means of thin layer chromatography. Three millilitres of absolute ethanol were added dropwise to $2 \mathrm{ml}$ of serum. The mixture was kept at $60^{\circ} \mathrm{C}$ for 15 minutes to allow proteins to precipitate. Negligible amounts of bile acid were trapped in the precipitate (recovery studies using labelled cholic acid showed less than $1.0 \%$ of the same acid in the lower phase). After centrifugation, $4 \mathrm{ml}$ of the above mixture was collected, dried under nitrogen stream, and redissolved in $200 \mathrm{ul}$ of a chloroform/methanol $(2 / 1$, $\mathrm{v} / \mathrm{v})$ mixture. One hundred ul were then run on precoated silica gel $G$ plates $(0.25 \mathrm{~mm}$ thick) (Merck, Darmstadt, GRF) using the II Hofmann (16) solvent system (propionic acid/isoamylacetate/ $\mathrm{H}_{2} \mathrm{O} /$ n-propanol: $75 / 100 / 25 / 50$ ).

$\mathrm{Rf}$ values were measured by using the respective conjugated and free bile acids standards.

The areas relative to the bile acids were then scraped and eluted with 400 ul of a chloroform/ methanol $(2 / 1, v / v)$ mixture.

After centrifugation, 200 ul were evaporated under nitrogen stream and redissolved in $100 \mathrm{ul}$ of phosphate buffer ( $\mathrm{pH} \mathrm{7.4).} \mathrm{Ten} \mathrm{to} 200$ ul were assayed by radioimmunoassay.

The data were expressed as umol/l and as the ratio between the glyco and tauro plus glyco bile acids conjugates.

\section{Results}

SERUM CHOLIC AND CHENODEOXYCHOLIC ACID FASTING LEVELS AND POSTPRANDIAL PEAKS Healthy subjects

In fasting conditions, chenodeoxycholic acid was the predominant bile acid in serum. It increased sharply after meals and the maximum postprandial rise occurred about 90 minutes after the meal. The postprandial rise in serum cholic acid occurred later (about 120 minutes) and was not so sharp and high as that of chenodeoxycholic acid.

The highest cholic and chenodeoxycholic acid serum rise was observed after the second meal; so these rises after the second meal $(p)$ were therefore chosen for comparison between controls and patients.

\section{Ileal resection}

Mean fasting levels of cholic and chenodeoxycholic acid did not differ from the controls (Fig. 1), even though two patients with ileal resection showed low $(0 \cdot 2$ umol/l) serum fasting cholic acid levels.

After meals, increases in cholic acid were lower than in the controls $(p<0.001)$ and sometimes even disappeared. Chenodeoxycholic acid postprandial levels were not significantly different from the controls, when all patients were considered together, but two populations of ileectomised patients were identified, one of which presented normal $(6 \cdot 6 \pm 1 \cdot 7 \mathrm{umol} / \mathrm{l})$ and one low $(1.9 \pm 0.4$ umol/l) chenodeoxycholic acid postprandial peaks (Fig. 1).

In the former group, the ascending colon had not been removed and four out of five patients had small ileal resections.
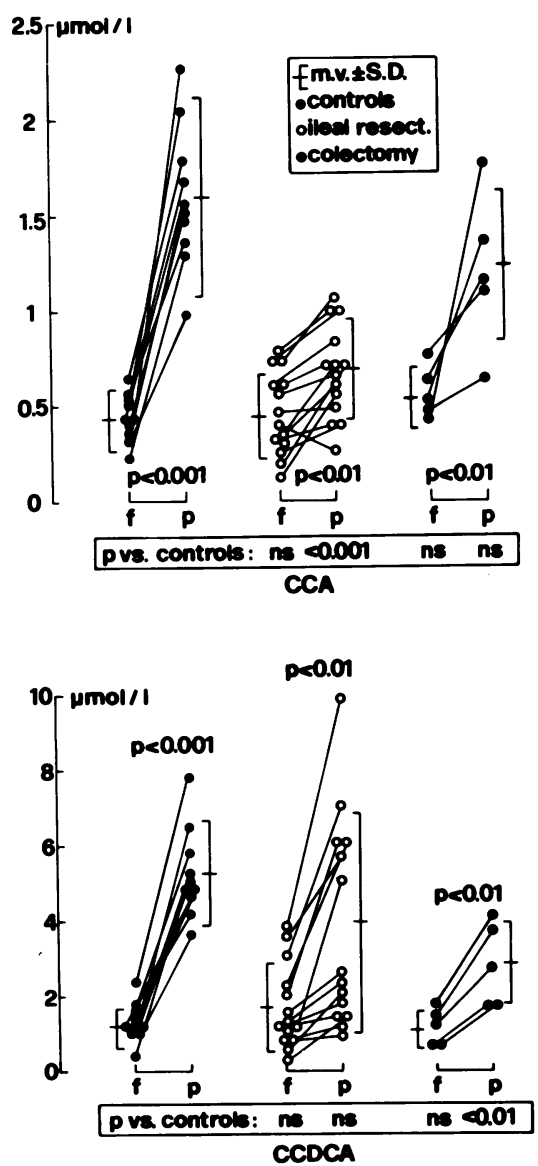

Fig. 1 Serum fasting levels $(f)$ and maximal postprandial peaks $(p)$ of cholic acid and chenodeoxycholic acid conjugates in the patients and controls. 
An inverse correlation was observed between serum cholic acid $(p<0.001)$ and chenodeoxycholic acid $(p<0.001)$ maximal postprandial increases and the daily faecal excretion of their respective forms and secondary bile acids (Fig. 2) in ileectomised patients.

\section{Colectomy}

Serum fasting levels of cholic and chenodeoxycholic acid were similar to the controls; maximum rises in cholic acid after food were not significantly different from the healthy subjects, while maximal rises in chenodeoxycholic acid after meals were lower $(\mathrm{p}<0 \cdot 01)$ compared with the controls (Fig. 1).
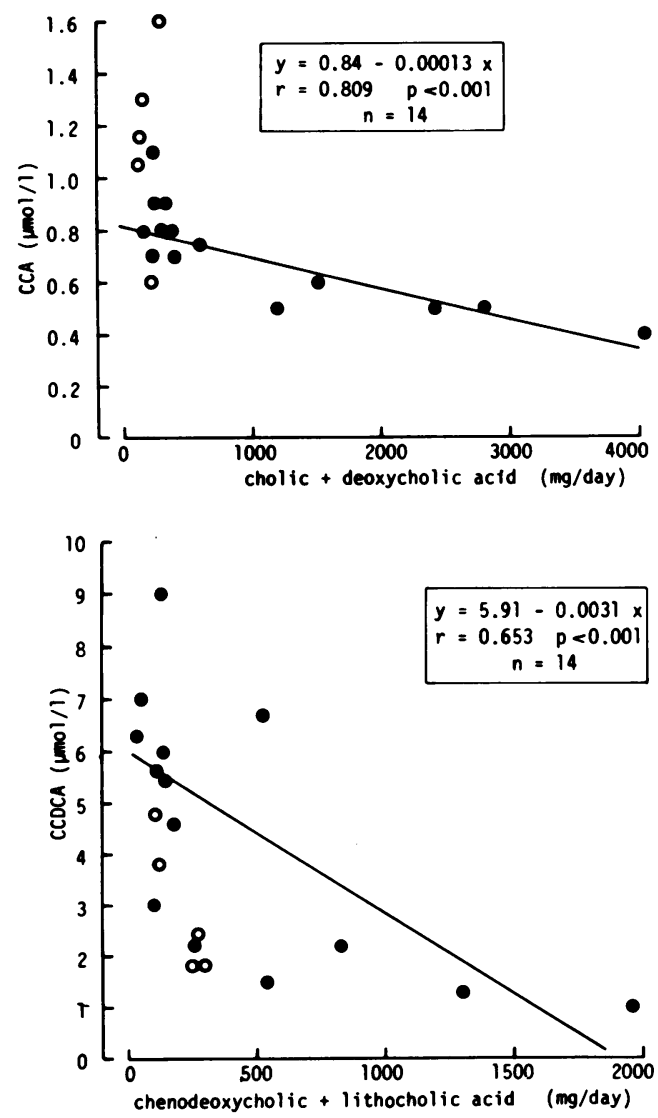

Fig. 2 Serum cholic acid maximal postprandial peaks plotted versus cholic + deoxycholic acid daily faecal excretion and serum chenodeoxycholic acid maximal postprandial peaks plotted versus chenodeoxycholic + lithocholic acid daily faecal excretion in the patients with ileal resections $(o)$ and patients with colectomy $(o)$. Only the patients with ileal resections were included in the statistical analysis.
Neither in patients with ileal resection nor colectomy were the serum cholic and chenodeoxycholic acid postprandial peaks reduced throughout the day.

\section{SERUM CHOLYL AND CHENYL CONJUGATES RATIO} (CHENODEOXYCHOLIC ACID/CHOLIC ACID + CHENODEOXYCHOLIC ACID)

In healthy subjects, no differences were found in the chenodeoxycholic acid/cholic acid+chenodeoxycholic acid ratio in the fasting and postprandial sera, chenodeoxycholic acid being the major bile acid in both conditions, while, in patients with ileal resection, the relative serum enrichment of chenylconjugates in the evening, compared with the morning $(p<0.01)$, resulted in an increase in the chenodeoxycholic acid/cholic acid+chenodeoxycholic acid ratio.

No differences were found between the colectomy patients and controls.

\section{SERUM GLYCINE/GLYCINE + TAURINE RATIO OF PRIMARY BILE ACIDS}

In healthy subjects, an increase in serum glycine conjugated cholic acid and a decrease in the taurine conjugated form explained a slight, not significant, increase in the $G / G+T$ ratio from the morning to the evening; chenodeoxycholic acid glycine and taurine conjugated forms did not differ throughout the day (Fig. 3).

In ileal resection, serum glycine conjugated cholic acid increased, while the serum taurine conjugated form either decreased or almost disappeared; the $\mathrm{G} / \mathrm{G}+\mathrm{T}$ ratio for cholic acid was significantly $(p<0.001)$ different in fasting conditions and after meal-stimulated enterohepatic recycling, but not significantly different from the controls in either conditions. The increase of the glycine conjugated chenodeoxycholic acid and the decrease of the taurine conjugated form accounted for a significant $(p<0 \cdot 001)$ increase in the $G / G+T$ ratio, which did not differ from the controls in fasting conditions, but was significantly $(p<0.01)$ different after meals.

Serum glycine and taurine conjugated primary bile acids were similar to the controls, both in fasting conditions and after meals in colectomy patients.

\section{Discussion}

In healthy subjects, the two primary bile acids are present in serum at low levels in fasting conditions and rise in response to meals. Increases in postprandial chenodeoxycholic acid are sharper and higher and occur earlier than those in cholic acid, possibly because the jejunum passively absorbs chenylconjugates better than cholylconjugates. ${ }^{17-19}$ 

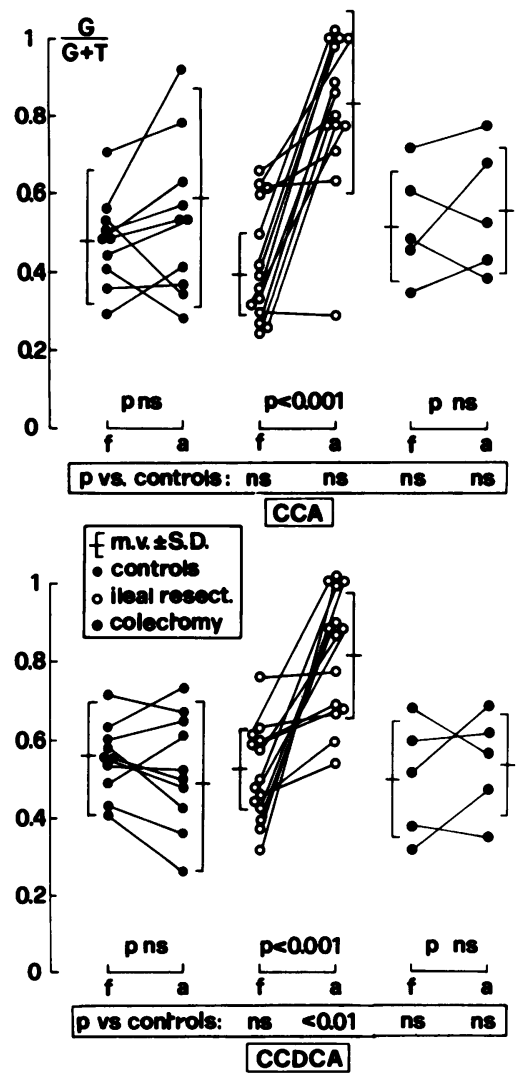

Fig. 3 Glycine/glycine + taurine conjugated cholic acid and chenodeoxycholic acid in fasting conditions $(f)$ and after meals (a) in patients with ileal resections, colectomy, and controls.

In patients with bile acid malabsorption, the mean serum fasting levels of cholic and chenodeoxycholic acid are not significantly lower then in the controls, though few patients present low cholic acid serum levels in fasting conditions.

These findings do not completely agree with a previous report ${ }^{\mathrm{T}}$ and seem to suggest that compensation mechanisms, like decreased transit rate of decreased bile acid load in the intestine, allow a more efficient absorption of both primary bile acids in fasting conditions than after meals, when transit time decreases and more bile acids reach the intestine after gall bladder emptying.

Both in the controls and in the patients, the highest bile acid postprandial peaks were found after the second meal, perhaps because of its higher caloric intake.

Schalm et $a l^{4}$ found lower, but constant rises in postprandial chenodeoxycholic acid, whereas cholic acid levels diminished progressively with each meal, consistent with a more severe malabsorption of cholyl, but not of chenyl, conjugates in patients with ileal resections.

The differences in the results obtained may be related to different experimental conditions (equicaloric liquid meals versus solid meals of different caloric loads). Data were not reported about the severity of the bile acid malabsorption in the patients studied in the above-mentioned investigation, and, therefore, it is possible that the two series of patients are not alike.

The present study confirms that two different enterohepatic circulatory dynamics exist for trihydroxy and dihydroxy bile acids, largely determined by the intestinal absorptions, as indicated by the serum bile acid levels; in cases of intestinal resection or disease, the interruption of the enterohepatic circulation of bile acids may be different for the two primary bile acids.

Serum bile acid levels give information about the presence of bile acid malabsorption, as indicated by the close correlation between postprandial levels of serum cholic and chenodeoxycholic acid and their respective forms in faeces (Fig. 2), in patients with documented bile acid malabsorption (positive cholyl-1- ${ }^{14} \mathrm{C}$ glycine test).

In the present paper, patients with large and small resections of the ileum showed a reduced postprandial peak of cholic acid, while patients with both large (four out of five) ileal resections and hemicolectomy presented reduced rises in chenodeoxycholic acid. These results confirm that cholic acid postprandial peaks may be a marker of ileal disease, while those of chenodeoxycholic acid provide indirect information about colonic impairment.

The ratio between chenyl and cholyl conjugates did not significantly change in the controls througout the day: on the contrary, patients with ileal resections showed a significant enrichment in serum chenyl conjugates compared with cholyl conjugates throughout the day. These changes, therefore, may be regarded as a test for bile acid malabsorption in patients with ileal disease. This test seems to be easy to perform, requiring the determination of cholic and chenodeoxycholic acid by radioimmunoassays on two serum samples only, obtained respectively in the morning and in the evening.

The progressive increase in the serum glycine conjugated compared with the taurine conjugated chenodeoxycholic acid in bile acid malabsorption additionally supports the idea that the integrity of the enterohepatic circulation of bile acids is partially restored by passive absorption, when the active one is impaired. 
References

1 La Russo NF, Körman MG, Hoffman NE, Hofmann AF. Dynamics of the enterohepatic circulation of bile acids. N Engl J Med 1974; 291: 689-92.

2 Hofmann AF, La Russo NF, Korman MG, et al. Physiological meaning and diagnostic sensitivity of serum cholate levels. In: Preising $\mathrm{R}$, Bircher $\mathrm{J}$, Paumgartner, eds. Liver. Aulendorf, Germany: Edito Cantor, 1976: 259-67.

3 Cowen AE, Korman MG, Hofmann AF, Thomas PJ. Plasma disappearance of radioactivity after intravenous ingestion of labelled bile acids in man. Gastroenterology 1975; 68: 1567-73.

4 La Russo NF, Hoffman NE, Korman MG, Hofmann AF, Cowen AE. Determinants of fasting and postprandial serum bile acid levels in healthy man. Dig Dis Sci 1978; 23: 385-91.

5 Erlinger S, Paupon R, Glasinovic J, Dumont $\mathbf{M}$. Hepatic uptake, storage and biliary transport maximum of bile acids in the dog. Bile acid metabolism in health and disease. In: Paumgartner G, Stiehl A, eds. Proceedings of 4th Bile Acid Meeting, Basel. Lancaster: MTP Press, 1977: 107-12.

6 Angelin B, Björkem I: Postprandial serum bile acids in healthy man. Evidence for differences in absorptive pattern between individual bile acids. Gut 1977; 18: 606-9.

7 Barbara L, Roda A, Roda E, et al. Diurnal variations of serum primary bile acids in healthy subjects and hepatobiliary disease patients. Rendic Gastroenterol 1976; 8: 194-8.

$8 \mathrm{Krag} \mathrm{E}$, Phillips SF. Active and passive bile acid absorption in man. Perfusion studies of the ileum and jejunum. J Clin Invest 1974; 53: 1686-94.

9 Angelin B, Einarsson K, Hellström K. Evidence for the absorption of bile acids in the proximal small intestine of normo and hyperlipidaemic subjects. Gut 1976; 17:
420-26.

10 Schalm SW, La Russo NF, Hofmann AF, Hoffman NE, Van Berge-Henegouwen GP, Korman MG. Diurnal serum levels of primary conjugated bile acids. Assessment by specific radioimmunoassays for conjugates of cholic and chenodeoxycholic acid. Gut 1978; 19: 1006-14.

11 Roda A, Roda E, Aldini R, et al. Development, validation and application of a single tube radioimmunoassay for cholic and chenodeoxycholic acid conjugates in human serum. Clin Chem 1977; 23: 2107-13.

12 Fromm H, Hofmann AF. Breath test for altered bile acid metabolism. Lancet 1971; 2: 621-5.

13 Roda A, Roda E, Aldini R, et al. Determination of ${ }^{14} \mathrm{CO}_{2}$ in breath and ${ }^{14} \mathrm{C}$ in stools after oral administration of cholyl $1-{ }^{14} \mathrm{C}$ glycine: clinical application. Clin Chem 1977; 23: 2127-32.

14 Fromm H, Thomas PJ, Hofmann AF. Sensitivity and specificity in tests of distal ileal function: prospective comparison of bile acid and vitamin $\mathbf{B}_{12}$ absorption in ileal resection patients. Gastroenterology 1973; 64: 1077-90.

15 Grundy SM, Ahrens EM Jr, Miettinen TA. Quantitative isolation and gas-liquid chromatographic analysis of total faecal bile acids. J Lipid Res 1965; 6: 397-410.

16 Hofmann AF. Thin layer absorption chromatography of free and conjugated bile acids on silicic acid. $J$ Lipid Res 1963; 2: 127-8.

17 Holt PR. Intestinal absorption of bile salts in the rat. Am J Physiol 1964; 207: 1.

18 Weiner IM, Lack L. Bile acid absorption: enterohepatic circulation. In: Code CF, Heidel W. Handbook of physiology. Section 6: Alimentary canal, vol 3. Baltimore: Williams and Wilkins, 1968: 1439-55.

19 Dietschy JM. Mechanism for intestinal absorption of bile acids. J Lipid Res 1968; 9: 297-309. 